\title{
SPATIAL STRUCTURES IN MAGNETIZABLE FLUIDS
}

\author{
N. F. Patsegon and L. N. Popova
}

UDC 532.528

\begin{abstract}
The conditions of existence and stability of spatial structures of ferroparticles of the type of isolated and periodic strata that are formed in fixed layers of magnetizable fluid in a constant homogeneous magnetic field are studied.
\end{abstract}

\section{Introduction}

Magnetizable fluids (magnetic fluids or ferrofluids) are artificial colloid solutions of ferroparticles with a characteristic size $\sim 10 \mathrm{~nm}$, coated with a layer of a surfactant, in a liquid carrier [14]. They are used as a lubricant in plain bearings, providing minimum friction in assemblies of drives of magnetic and optical disks, sealants that protect the body of a hard disk of a computer from dust, acoustic loudspeakers for damping a membrane, separators, and accelerometers, and find extensive use in modern high technologies [13, 20, 23].

In works published in recent years $[16,19,22]$, a new field of possible application of magnetizable fluids, namely, magnetooptics, has been discussed. Experiments with films of these fluids show that, under the action of a magnetic field, ferroparticles that enter into the fluid form structures, the configuration of which depends on the direction of the field and its value, the fluid becomes anisotropic, and, correspondingly, the conditions of its interaction with optical radiation change. This property of magnetizable fluids can be used for the development of optical gates, light modulators, controlled filters, diffraction grating, and displays [13, 22].

New possibilities of application of magnetizable fluids are stimulated by theoretical and experimental investigation of processes occurring with ferroparticles in magnetic fields (see [2] and references in it).

The change in the microstructure of magnetizable fluids is caused by the possibility of formation of aggregates consisting of a finite number of ferroparticles in it $[1,15]$. In [6], an appropriate model of a medium with a changing microstructure, which describes processes of formation and breakdown of aggregates of ferroparticles, was proposed. The state variables of this medium are the mean number of particles in aggregates $\gamma=v^{-1}$ and the parameter of the effective magnetic field $\lambda$ that takes into account the field induced by ferroparticles. In this case, the change in the magnetic state of the medium is set by a system of two quasilinear equations of parabolic type for $\lambda$ and $v$. In [12], a qualitative investigation of a concentrated dynamic system that exists in the limiting case, when diffusion processes in a fluid are negligibly small, was performed. Homogeneous equilibrium states of a medium were considered in $[8,21]$. In these works, equilibrium magnetization curves of the fluid were constructed, and the conditions of existence of wave modes of the type of switching waves were obtained. In [9-11, 21], spatial and spatial-time structures of the type of static and running autosolitons, which form in the volume of a magnetizable fluid in a constant homogeneous magnetic field, were studied. They are realized in the form of parallel layers, strips, and cylindrical columns, oriented along the field, in which aggregates of ferroparticles are concentrated. As a result of a comparison of calculated and experimentally measured characteristics of the structures, estimates of the parameters of the model of the medium were obtained.

Karazin Kharkiv National University, Kharkiv, Ukraine.

Translated from Matematychni Metody ta Fizyko-Mekhanichni Polya, Vol. 53, No. 3, pp. 159-169, July-September, 2010. Original article submitted November 27, 2009. 
In the present work, we consider the problem of stability of spatial structures of the type of isolated and periodic strata, which is important for practical applications. In the investigation of the stability, the general theory of autosolitons, the results and methods of which were presented in the monograph [4], is used.

\section{Stationary Structures in a Magnetizable Fluid}

Let us consider a layer of fluid of thickness $\ell$, located between two parallel infinite nonmagnetic plates, in a constant homogeneous magnetic field parallel to the layer. We choose the origin $O$ of a Cartesian rectangular coordinate system at the center of the layer and direct the axis $O x$ perpendicularly to the layer, and the axis $O y$ parallel to the magnetic field strength. To describe the behavior of the fluid, we use a model of a medium with a changing microstructure [6]. The equilibrium magnetization of this medium is determined by the density of the medium $\rho$, temperature $T$, the magnetic field strength $H$, the mean number of particles in aggre-

gates $\gamma=v^{-1}$, and the parameter of the effective magnetic field $\lambda$. The magnetic-dipole interaction between ferroparticles in the approximation of the self-consistent field is taken into account via the parameter $\lambda$. The strength of the magnetic field that acts on the liquid volume is represented in the form $H_{e}=H+\lambda M$, where $\lambda M$ is the strength of the field induced by particles. The change of the magnetic state of the medium is described by a system of equations, which, in the one-dimensional case, has the form

$$
\tau_{\lambda} \frac{\partial \lambda}{\partial t}=D_{\lambda} \frac{\partial^{2} \lambda}{\partial x^{2}}+Q^{(\lambda)}(\lambda, v ; H), \quad \tau_{v} \frac{\partial v}{\partial t}=D_{v} \frac{\partial^{2} v}{\partial x^{2}}+Q^{(v)}(\lambda, v ; H)
$$

The functions $Q^{(\lambda)}(\lambda, v ; H)$ and $Q^{(v)}(\lambda, v ; H)$ are set by the expressions

$$
\begin{gathered}
Q^{(\lambda)}(\lambda, v ; H)=\frac{1}{2}\left[L^{2}-L_{*}^{2}-2 \beta\left(\lambda-\lambda_{1 *}\right)\left(\lambda-\lambda_{2 *}\right)\left(\lambda-\lambda_{3^{*}}\right)\right], \\
Q^{(v)}(\lambda, v ; H)=f(\xi)-f\left(\xi_{*}\right)+\alpha\left(v-v_{1 *}\right)\left(v-v_{2 *}\right)\left(v-v_{3 *}\right) .
\end{gathered}
$$

Here, $\xi=m_{1} H_{e} /(k T v), n_{1}=c_{1} \rho / \mathcal{M}$ is the volume density of ferroparticles, $c_{1}$ is the mass concentration of particles, which is assumed constant, $\mathcal{M}$ is the mass of an isolated ferroparticle, and $k$ is the Boltzmann constant. The magnetization of the fluid is determined by the equation $M=M_{s} L(\xi)$, where $L(\xi)=\operatorname{coth} \xi-1 / \xi$ is the Langevin function, $M_{*}$ is the magnetization of structurization of the fluid, $M_{*}=M_{s} L\left(\xi_{*}\right)=M_{s} L_{*}, \quad M_{s}=m_{1} n_{1}$ is the saturation magnetization of the fluid, $\lambda_{i^{*}}, \quad v_{i *}, i=1,2,3$, are the equilibrium values of $\lambda$ and $v$ for $M=M_{*}, \lambda_{1 *}>\lambda_{3 *}>\lambda_{2 *}, \lambda_{1 *}+\lambda_{2 *}=2 \lambda_{3 *}, v_{1 *}>v_{3 *}>v_{2 *}$,

$$
v_{i *}-v_{j *}=m_{1} M_{s} L_{*} \cdot\left(\lambda_{i *}-\lambda_{j *}\right) /\left(k T \xi_{*}\right), \quad \alpha=\left(k T /\left(m_{1} M_{s}\right)\right)^{3} \cdot\left(\xi_{*} / L_{*}\right)^{4} \beta,
$$

and

$$
f(\xi)=\ln (\sinh \xi / \xi)-\xi L(\xi) .
$$

The parameters $\tau_{\lambda}, \tau_{v}, D_{\lambda}, D_{v}$, and $\beta$ are assumed to be constant. 
The homogeneous equilibrium states of the fluid $\lambda=$ const and $v=$ const satisfy the equations

$$
Q^{(\lambda)}(\lambda, v ; H)=0, \quad Q^{(v)}(\lambda, v ; H)=0 .
$$

For $\lambda_{1 *} \neq \lambda_{2 *}$, the considered medium is characterized by a smooth criss-cross magnetization curve and, in different intervals of change in the magnetic field strength, has from one to nine equilibrium states [21]. System (1) differs in this from monostable and bistable systems, for which the theory of autosolitons was constructed in [4].

We investigate the case of the K-system [4], for which $D_{v} \ll D_{\lambda}$. In the K-system, the characteristic scale of change in the parameter of the effective magnetic field in the space substantially exceeds the characteristic scale of change in the mean number of particles in aggregates, i.e., the parameter $v$ is a fast variable, and the parameter $\lambda$ is a slow variable. Moreover, we assume that the following condition holds:

$$
D_{v} \ll \ell^{2} \ll D_{\lambda}
$$

The change in the magnetic state of the medium under condition (3) is described by the following system of equations (if terms of order $\ell^{2} / D_{\lambda}$ are neglected):

$$
\begin{gathered}
\tau_{v} \frac{\partial v}{\partial t}=D_{v} \frac{\partial^{2} v}{\partial x^{2}}+Q^{(v)}(\lambda, v ; H), \\
\tau_{\lambda} \frac{\partial \lambda}{\partial t}=\frac{1}{\ell} \int_{-\ell / 2}^{\ell / 2} Q^{(\lambda)}(\lambda, v ; H) d x .
\end{gathered}
$$

The second equation was obtained by averaging the first equation of system (1) over the thickness of the layer of the fluid.

Let us find stationary solutions of the system of equations (4), (5) that satisfy the boundary conditions which correspond to the absence of flows of the parameters $\lambda$ and $v$ through the boundary

$$
\frac{d \lambda}{d x}\left(-\frac{\ell}{2}\right)=\frac{d \lambda}{d x}\left(\frac{\ell}{2}\right)=0, \quad \frac{d v}{d x}\left(-\frac{\ell}{2}\right)=\frac{d v}{d x}\left(\frac{\ell}{2}\right)=0
$$

They are solutions of the boundary problem

$$
\begin{gathered}
D_{v} v^{\prime \prime}+Q^{(v)}\left(\lambda_{s}, v(x) ; H\right)=0, \\
\frac{d v}{d x}\left(-\frac{\ell}{2}\right)=\frac{d v}{d x}\left(\frac{\ell}{2}\right)=0, \\
\int_{-\ell / 2}^{\ell / 2} Q^{(\lambda)}\left(\lambda_{s}, v(x) ; H\right) d x=0,
\end{gathered}
$$


where $\lambda=\lambda_{s}=$ const. In the considered case, the equations of electrodynamics and boundary conditions for the magnetic field are automatically satisfied.

In [10], it was shown that there exists a range of values of the magnetic field strength in which the curve of local coupling determined by the equation $Q^{(v)}(\lambda, v ; H)=0$ for $H=$ const is $И$-shaped, i.e., the considered system is an И-system [4]. The plot of the dependence of $Q^{(v)}(\lambda, v ; H)$ on $v$ for a fixed value of $\lambda$ reproduces qualitatively the curve of the local coupling and is also $И$-shaped.

The phase portrait of the dynamic system to which Eq. (7) is reduced depends on the quantity $\lambda_{s}$. If $\lambda_{-}<\lambda_{s}<\lambda_{+}$, where $\lambda_{-}$and $\lambda_{+}$are the minimum and maximum values of the function $\lambda(v)$, which sets the curve of the local coupling, the system has the following three fixed points (denote them by $v_{1}, v_{2}$, and $\left.v_{3}\left(v_{1}<v_{2}<v_{3}\right)\right)$ : the saddles $v_{1}, v_{3}$, and the center $v_{2}$. For the value of $\lambda_{s}$ that satisfies the condition

$$
\int_{v_{1}}^{v_{3}} Q^{(v)}\left(\lambda_{s}, v ; H\right) d v=0
$$

the phase portrait contains a cell bounded by two heteroclinic trajectories, namely, separatrices, one of which emanates from the saddle $v_{1}$ and enters into the saddle $v_{3}$, and the other, vice versa, emanates from the saddle $v_{3}$ and enters into the saddle $v_{1}$. The closed trajectory close to the boundary of the cell corresponds to a wide stratum at the center of the layer of the fluid. The shape of the stratum contains segments of smooth and abrupt change in the parameter $v$ : on the plane $(\lambda, v)$, smooth changes in $v$ occur in small neighborhoods of the points $\left(\lambda_{s}, v_{1}\right)$ and $\left(\lambda_{s}, v_{3}\right)$, and the abrupt changes take place along the segment $v_{1}<v_{2}<v_{3}$ of the straight line $\lambda=\lambda_{s}$.

To construct the shape of the stratum, we approximate the function $Q^{(v)}\left(\lambda_{s}, v ; H\right)$ by a cubic polynomial that has the same roots $v_{1}, v_{2}, v_{3}$ and nearly the same derivatives at the points $v_{1}, v_{2}$, and $v_{3}$

$$
\begin{gathered}
Q^{(v)}\left(\lambda_{s}, v ; H\right) \approx Q(v)=-B\left(v-v_{1}\right)\left(v-v_{2}\right)\left(v-v_{3}\right), \quad B>0 \\
\sum_{i=1}^{3} \frac{\partial Q^{(v)}\left(\lambda_{s}, v_{i} ; H\right)}{\partial v}=\left.\sum_{i=1}^{3} \frac{d Q}{d v}\right|_{v=v_{i}} .
\end{gathered}
$$

The constant $B$ is determined from the last condition

$$
B=-\frac{\sum_{i=1}^{3} \frac{\partial Q^{(v)}\left(\lambda_{s}, v_{i} ; H\right)}{\partial v}}{\left(v_{2}-v_{1}\right)^{2}+\left(v_{3}-v_{1}\right)\left(v_{3}-v_{2}\right)}
$$

The equation $D_{v} v^{\prime \prime}+Q(v)=0$ has the solutions

$$
v(x)=\frac{v_{1}+v_{3} \exp \left[ \pm \sqrt{B /\left(2 D_{v}\right)}\left(v_{3}-v_{1}\right) x\right]}{1+\exp \left[ \pm \sqrt{B /\left(2 D_{v}\right)}\left(v_{3}-v_{1}\right) x\right]},
$$




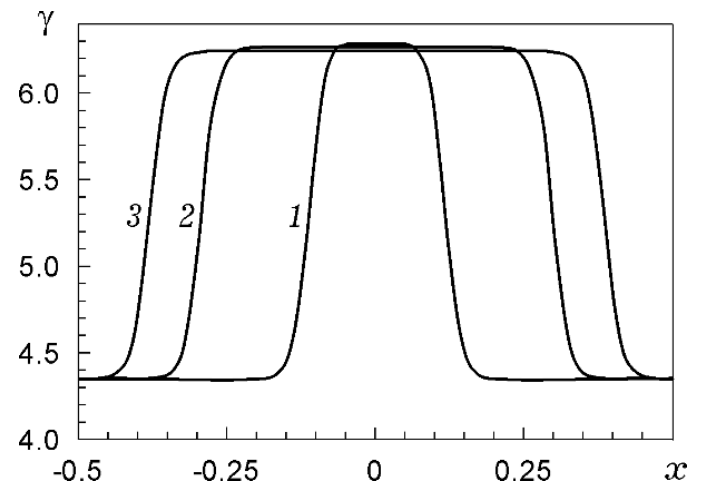

(a)

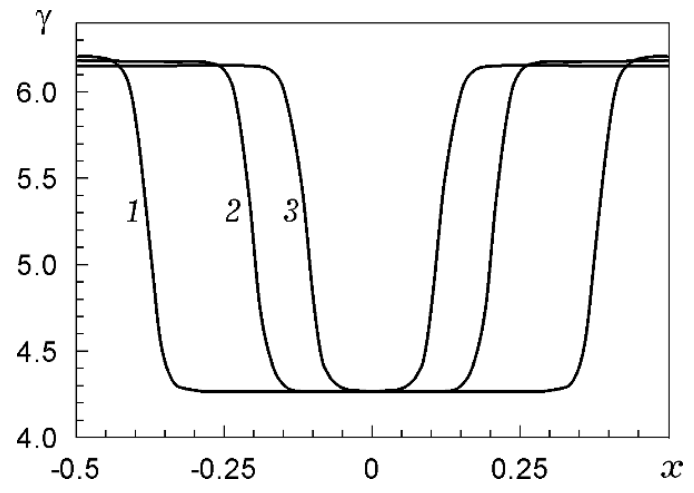

(b)

Fig. 1. Wide strata in the magnetizable fluid: curves $1-3$ correspond to $H=15,18,21 \mathrm{Oe} ; \gamma$ is the number of particles in aggregates.

which correspond to the separatrices of the saddle points. Joining these solutions at the center of the segment $-\ell / 2<x<\ell / 2$, we obtain an approximate expression for the wide stratum at the center of the layer of the fluid. For a hot (cold) stratum, the mean number of particles in aggregates at the center of the segment is larger (smaller) than that near the boundary. Replacing the stratum by a step function, from the integral condition (9) we can determine its size

- for a hot stratum

$$
\ell_{s}=\frac{Q^{(\lambda)}\left(\lambda_{s}, v_{1} ; H\right)}{Q^{(\lambda)}\left(\lambda_{s}, v_{1} ; H\right)-Q^{(\lambda)}\left(\lambda_{s}, v_{3} ; H\right)} \ell,
$$

- for a cold stratum

$$
\ell_{s}=\frac{Q^{(\lambda)}\left(\lambda_{s}, v_{3} ; H\right)}{Q^{(\lambda)}\left(\lambda_{s}, v_{3} ; H\right)-Q^{(\lambda)}\left(\lambda_{s}, v_{1} ; H\right)} \ell
$$

A solution of the wide-stratum type exists only in the case where the expression that sets its size is positive.

In Fig. 1, we show strata that form in a layer of a magnetizable fluid at different values of the magnetic field strength (Fig. 1a shows a hot stratum, and Fig. 1b shows a cold stratum). Calculations were performed for the following values of the parameters of the model: $m_{1}=10^{-16} \mathrm{erg} / \mathrm{gf}, M_{s}=40 \mathrm{gf}, \xi_{*}=1, \beta=-0.1, \lambda_{1 *}=5$, $\lambda_{3 *}=4$, and $v_{3 *}=0.2$.

In the indicated range of the magnetic field strength, with increase in $H$, the width of the hot stratum increases, and the width of the cold stratum decreases. In this case, the height of the stratum $\Delta \gamma=1 / v_{3}-1 / v_{1}$ changes insignificantly and agrees with an estimate for the number of particles in chain aggregates presented in [18]. In Fig. 2, we show the corresponding distributions of the magnetization of the medium.

Along with a singular hot and a cold stratum, problem (7)-(9) has a set of periodic solutions in the form of a sequence of identical strata of period $L_{p}=\ell / N \quad(N$ is a natural number). These solutions describe periodic structures in the form of layers of the more or less aggregated medium in the layer of the fluid that are equidistant from one another. 


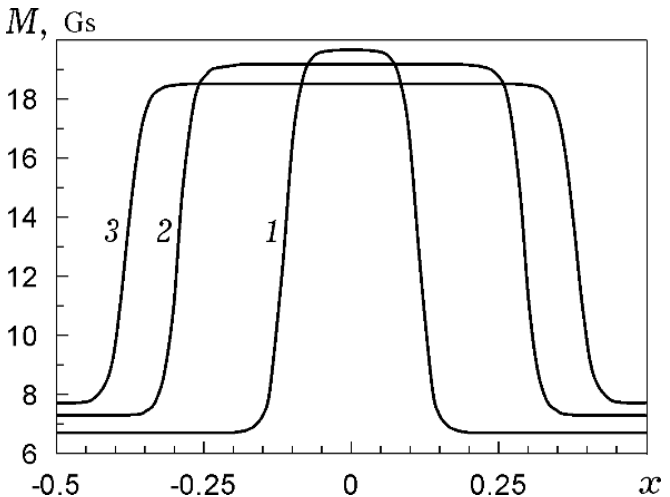

(a)

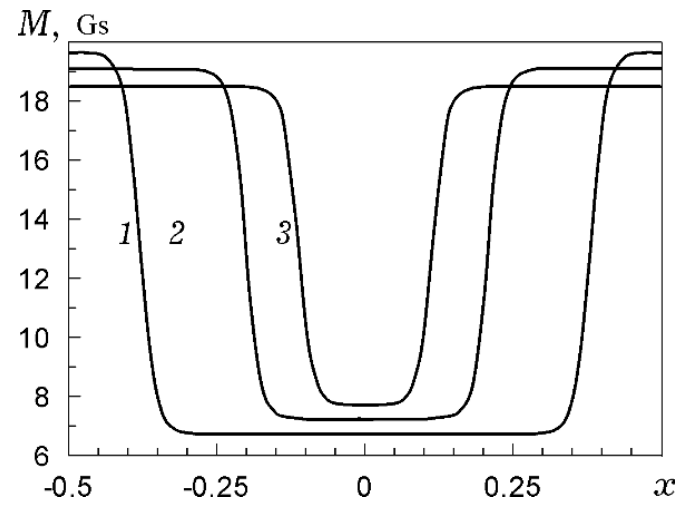

(b)

Fig. 2. Distribution of the magnetization in the layer of the fluid: curves $1-3$ correspond to $H=15,18,21$ Oe.

The solutions of system (7)-(9) model the formation of stationary structures in the fluid also in the case where the axis $O x$ is parallel to the layer. They are realized in the form of one or several strips oriented along the magnetic field in which aggregates of ferroparticles are concentrated.

The strip structures of ferroparticles are observed experimentally in films of magnetizable fluids [17, 19, 22]. The authors of [17] used a kerosene-based ferrofluid with magnetite particles stabilized by oleic acid in experiments. A layer of the fluid with a thickness of several micrometers was deposited on the bottom of a closed glass rectangular cell and placed in a magnetic field. Observations with an optical microscope showed that within $10 \mathrm{sec}$ after switching a field parallel to the layer of the fluid, initially randomly distributed ferroparticles are arranged in needle-like chains oriented along the field; with time, aggregates become longer and thicker, and within $2 \mathrm{~h}$, a quasiperiodic strip structure of nanoparticles with a width of strips $\sim 1 \mu \mathrm{m}$ and a distance of $\sim 20 \mu \mathrm{m}$ between them forms. Similar experiments were described in [13, 19, 22].

In $[7,11]$, a comparison of computed characteristics of stationary structures in a layer of a magnetizable fluid with experimental data was performed, as a result of which the following estimates for the parameters of a model of a medium with a changing microstructure were obtained: $\tau_{v} \sim 10 \mathrm{sec}, \tau_{\lambda} \sim 7200 \mathrm{sec}, D_{\lambda} \sim 2 \mu \mathrm{m}^{2}$, and $D_{v} \ll 1 \mu \mathrm{m}^{2}$.

\section{Stability of Stationary Structures}

Let us consider the stability of the stationary solution $v=v(x), \quad \lambda=\lambda_{s}=$ const of system (4), (5) for relatively small perturbations.

In dimensionless variables, Eqs. (4) and (5) have the form

$$
\frac{\partial v}{\partial t}=\frac{\partial^{2} v}{\partial x^{2}}+Q^{(v)}(\lambda, v ; H), \quad \alpha^{-1} \frac{\partial \lambda}{\partial t}=\left\langle Q^{(\lambda)}(\lambda, v ; H)\right\rangle
$$

Here, we use $\sqrt{D_{v}}$ as a characteristic linear size and $\tau_{v}$ as a characteristic time; we retain the same notation for dimensionless variables $x$ and $t$ as earlier for dimensional variables $\alpha:=\tau_{v} / \tau_{\lambda}$; the angle brackets mean averaging over the dimensionless thickness of the layer of the fluid. 
Let us represent perturbations in the form

$$
\delta v(x, t)=\delta v(x) \exp (-\eta t), \quad \delta \lambda(t)=\delta \lambda \exp (-\eta t)
$$

where $\delta v(x)$ satisfies the cyclic boundary conditions.

The investigation of the stability of the inhomogeneous distribution $v=v(x), \lambda=\lambda_{s}=$ const is reduced to the problem for eigenvalues

$$
\begin{gathered}
(A-\eta) \delta v=Q_{\lambda}^{(v)}\left(\lambda_{s}, v(x) ; H\right) \delta \lambda, \\
\delta \lambda=\left(\mu-\eta \alpha^{-1}\right)^{-1}\left\langle Q_{v}^{(\lambda)} \delta v\right\rangle \\
\delta v\left(-\frac{\ell}{2}\right)=\delta v\left(\frac{\ell}{2}\right), \quad \frac{d \delta v}{d x}\left(-\frac{\ell}{2}\right)=\frac{d \delta v}{d x}\left(\frac{\ell}{2}\right),
\end{gathered}
$$

where

$$
A:=-\frac{d^{2}}{d x^{2}}-Q_{v}^{(v)}\left(\lambda_{s}, v(x) ; H\right), \quad \mu:=-\left\langle Q_{\lambda}^{(\lambda)}\left(\lambda_{s}, v(x) ; H\right)\right\rangle .
$$

The solution $v=v(x), \lambda=\lambda_{s}$ is stable if $\operatorname{Re} \eta>0$, otherwise, it is unstable.

In contrast to systems considered in [4], for the magnetizable fluid, the parameter $\mu$ can take both positive and negative values.

In a medium whose magnetic state is determined by only one variable $v$, for $\lambda=$ const and, correspondingly, $\delta \lambda=0$, the inhomogeneous distributions $v=v(x)$ are unstable. In this case, the investigation of stability is reduced to the determination of the eigenvalues $\eta=p_{n}$ of the differential equation

$$
A \delta v=p \delta v
$$

with the boundary conditions (14). It follows from the oscillation theorem [3,5] that, for this problem, there exists an infinite set of eigenvalues, and all eigenvalues are real and can be arranged in the form of the infinitely increasing sequence $p_{0}<p_{1}<p_{2}<\ldots$, and the eigenfunction $\delta v_{n}$, which corresponds to the eigenvalue $p_{n}$, has exactly $n$ zeros in the interval $-\ell / 2<x<\ell / 2$. The differentiation of the first equation of system (10) with respect to $x$ in the stationary case shows that $d v / d x$ is the eigenfunction of the operator $A$, which corresponds to a zero eigenvalue [4]. The function $v(x)$, which determines the shape of the stratum, attains an extremum at the point $x=0$, and, therefore, the derivative $d v / d x$ has a zero at this point, i.e., it is not an eigenfunction of $\delta v_{0}$, and $\eta=p_{0}<0$.

Consider the general case where $\delta \lambda \neq 0$. Substituting the expression for $\delta \lambda$ from (13) in (12), we get

$$
(A-\eta) \delta v=Q_{\lambda}^{(v)}\left\langle Q_{v}^{(\lambda)} \delta v\right\rangle\left(\mu-\eta \alpha^{-1}\right)^{-1}
$$


The expansion of $\delta v(x)$ in series in terms of the eigenfunctions $\delta v_{n}$ of problem (15), (14)

$$
\delta v(x)=\sum_{n=0}^{\infty} c_{n} \delta v_{n}(x)
$$

and the substitution of this expansion into (16) leads to the following system of linear homogeneous equations for the coefficients $c_{i}$ :

$$
c_{i}\left(p_{i}-\eta\right)+\sum_{n=0}^{\infty} c_{n}\left(\eta \alpha^{-1}-\mu\right)^{-1}\left\langle Q_{\lambda}^{(v)} \delta v_{i}\right\rangle\left\langle Q_{v}^{(\lambda)} \delta v_{n}\right\rangle=0, \quad i=0,1, \ldots
$$

Here, we take into account that the system of the functions $\delta v_{n}$ is orthonormal.

Let us introduce the notation

$$
a_{i}:=-\frac{1}{\mu}\left\langle Q_{\lambda}^{(v)} \delta v_{i}\right\rangle\left\langle Q_{v}^{(\lambda)} \delta v_{i}\right\rangle
$$

In the considered case,

$$
Q_{v}^{(\lambda)}=\frac{k T}{m_{1} M_{s}} Q_{\lambda}^{(v)}
$$

and, therefore,

$$
a_{i}=-\frac{1}{\mu} \frac{k T}{m_{1} M_{s}}\left(\left\langle Q_{\lambda}^{(v)} \delta v_{i}\right\rangle\right)^{2}
$$

i.e., all values of $a_{i}$ have the equal signs opposite to the sign of $\mu$. They can be expressed in terms of the positive values of $b_{i}$ :

$$
a_{i}=-\frac{1}{\mu} b_{i}
$$

which are determined as follows:

$$
b_{i}=\frac{k T}{m_{1} M_{s}}\left(\left\langle Q_{\lambda}^{(v)} \delta v_{i}\right\rangle\right)^{2}
$$

where $b_{i}=0$ for odd $i$ because $\delta v_{i}$ are odd functions, $Q_{\lambda}^{(v)}\left(\lambda_{s}, v(x) ; H\right)$ is an even function, and $b_{i}>0$ for even $i$. 
Taking into account (20), we write system (18) in the form

$$
\left[p_{i}-\eta+\left(\eta \alpha^{-1}-\mu\right)^{-1} b_{i}\right] c_{i}+\sum_{n=0}^{\infty}{ }^{\prime}\left(\eta \alpha^{-1}-\mu\right)^{-1}\left\langle Q_{\lambda}^{(v)} \delta v_{i}\right\rangle\left\langle Q_{v}^{(\lambda)} \delta v_{n}\right\rangle c_{n}=0, \quad i=0,1, \ldots
$$

The equality of the determinant of this system to zero gives the following equation for the determination of $\eta[4]:$

$$
\left[1-\sum_{n=0}^{\infty} b_{n}\left(p_{n}-\eta\right)^{-1}\left(\mu-\eta \alpha^{-1}\right)^{-1}\right] \prod_{n=0}^{\infty}\left(p_{n}-\eta\right)=0
$$

If the function $v(x)$ describes a periodic function in the volume of the fluid containing two or more strata, then its derivative $d v / d x$ has more than one root in the segment $(-\ell / 2, \ell / 2)$. In this case, Eq. (21) is satisfied for $\eta=p_{1}<0$, and the structure is unstable.

Consider the distribution of $v(x)$ in the form of a stratum. In this case, the derivative $d v / d x$ has a root, and to it there corresponds the eigenfunction $\delta v_{1}$, which corresponds to a zero eigenvalue $p_{1}=0$. Hence, $p_{0}<0$, and, for $n \geq 2$, all eigenvalues $p_{n}$ are positive.

Equation (21) is satisfied for $\eta=p_{n}, n \geq 1$, or in the case where the expression in brackets becomes zero. The values $\eta=p_{n} \geq 0$ for $n \geq 1$ are not dangerous from the viewpoint of loss of stability. Therefore, the stability of the stratum is determined by the values of $\eta$ that make the brackets in (21) vanish.

If we introduce the notation $\eta=i \omega$, the condition of stability takes the form $\operatorname{Im} \omega<0$. The stratum is unstable if the function in brackets in Eq. (21)

$$
D(\omega):=1-\sum_{n=0}^{\infty} b_{n}\left(p_{n}-i \omega\right)^{-1}\left(\mu-i \omega \alpha^{-1}\right)^{-1}
$$

has zero in the upper half-plane $\omega(\operatorname{Re} \omega, \operatorname{Im} \omega)$.

The real part of $D(\omega)$ is set by the equation

$$
\operatorname{Re} D(\omega)=1+\frac{\alpha}{\omega^{2}+\alpha^{2} \mu^{2}} \sum_{n=0}^{\infty} \frac{\omega^{2}-\mu \alpha p_{n}}{p_{n}^{2}+\omega^{2}} b_{n}
$$

and is an even function of $\omega$, and $\operatorname{Re} D( \pm \infty)=1$.

The imaginary part of $D(\omega)$ is set by the expression

$$
\operatorname{Im} D(\omega)=-\frac{\alpha \omega}{\omega^{2}+\alpha^{2} \mu^{2}} \sum_{n=0}^{\infty} \frac{\mu \alpha+p_{n}}{p_{n}^{2}+\omega^{2}} b_{n}
$$

it is an odd function of $\omega$, and, furthermore, $\operatorname{Im} D(0)=0$ and $\operatorname{Im} D( \pm \infty)=0$. The value of the derivative of this function at the point $\omega=0$ 


$$
\left.\frac{d}{d \omega} \operatorname{Im} D(\omega)\right|_{\omega=0} \approx-\frac{b_{0}}{\mu p_{0}^{2}}
$$

is negative for $\mu>0$ and positive for $\mu<0$.

The function $\operatorname{Im} D(\omega)$ can vanish for nonzero finite values of $\omega$. Let us denote this root, if it exists, by $\omega_{1}$. It follows from (24) that

$$
b_{0}=-\frac{p_{0}^{2}+\omega_{1}^{2}}{\alpha \mu+p_{0}} \sum_{n=2}^{\infty} \frac{\alpha \mu+p_{n}}{p_{n}^{2}+\omega_{1}^{2}} b_{n} .
$$

This equality is possible only in the case where $\alpha \mu+p_{0}<0$, i.e., for $\alpha \mu<-p_{0}$. Substituting the value for $b_{0}$ from (26) into (23), we obtain

$$
\operatorname{Re} D\left(\omega_{1}\right)=1-\frac{\alpha}{\alpha \mu+p_{0}} \sum_{n=2}^{\infty} \frac{p_{n}-p_{0}}{p_{n}^{2}+\omega_{1}^{2}} b_{n}>1 .
$$

In [4], it was shown that $p_{0}$ is a small negative value, and, hence,

$$
D(0)=1-\frac{b_{0}}{\mu p_{0}}-\frac{1}{\mu} \sum_{n=2}^{\infty} \frac{b_{n}}{p_{n}} \approx-\frac{b_{0}}{\mu p_{0}} .
$$

Let us use the argument principle for the analysis of the function $D(\omega)$

$$
N=P+\frac{1}{2 \pi} \Delta \arg D(\omega)
$$

where $N$ and $P$ are the numbers of zeros and poles of the function $D(\omega)$ in the upper half-plane.

It can be seen from expression (22) that $D(\omega)$ has poles at the points $\omega=-i p_{n}, \quad n=0,2,4, \ldots$, and $\omega=-i \mu \alpha$; the poles $\omega=-i p_{n}$ for $n \neq 0$ are located in the lower half-plane.

Let $\mu>0$. It follows from (25) and (28) that $D(0)>1, d(\operatorname{Im} D(\omega)) /\left.d \omega\right|_{\omega=0}<0$, and that the function $D(\omega)$ has only one pole $\omega=-i p_{0}$ in the upper half-plane, i.e., $P=1$. In Fig. 3, we show the behavior of the function $D(\omega)$ in the traversal of the upper half-plane: in Fig. 3a, the function $\operatorname{Im} D(\omega)$ does not have nonzero finite toots; in Fig. 3b, the function $\operatorname{Im} D(\omega)$ has one such root. In both cases, $\Delta \arg D(\omega)=0, N=1$, and the stratum is unstable. If the function $\operatorname{Im} D(\omega)$ has several roots, then since, for each of them, $\operatorname{Re} D(\omega)>1$, they do not influence the value of $\Delta \arg D(\omega)$.

Let $\mu<0$. In this case, $D(0)<0$, and the function $D(\omega)$ has two poles in the upper half-plane $\omega=$ $-i p_{0}$ and $\omega=-i \mu \alpha$, i.e., $P=2$. The behavior of $D(\omega)$ in the traversal of the upper half-plane is shown in Fig. 4. In Fig. 4a, the function $\operatorname{Im} D(\omega)$ does not have nonzero finite roots, and, in Fig. 4b, the function $\operatorname{Im} D(\omega)$ has one such root. If $\mu<0$, then $\Delta \arg D(\omega)=-2 \pi$, i.e., as follows from (29), $N=1$, and the stratum is unstable. If the function $\operatorname{Im} D(\omega)$ has several roots, then, since, for each of them, $\operatorname{Re} D(\omega)>1$, they do not affect the value of $\Delta \arg D(\omega)$. 


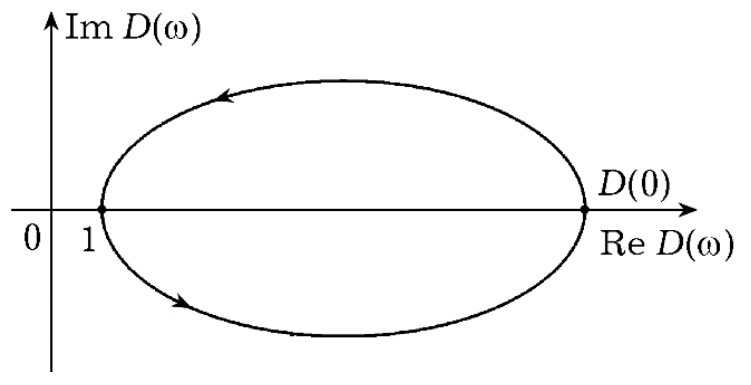

(a)

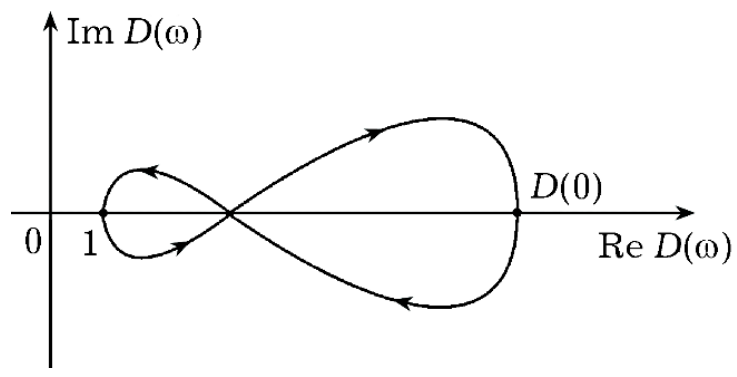

(b)

Fig. 3. Behavior of the function $D(\omega)$ in the traversal of the upper plane for $\mu>0$.

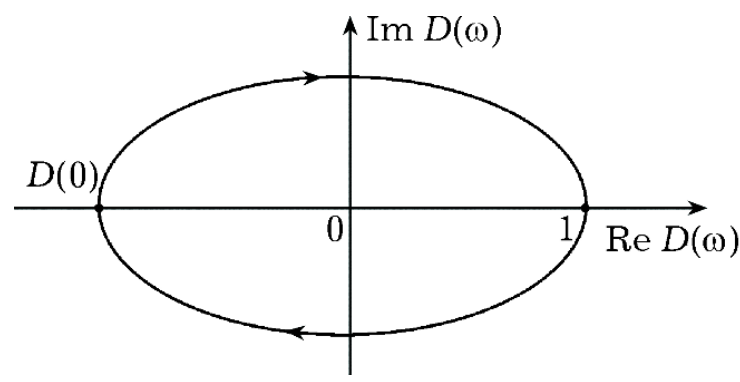

(a)

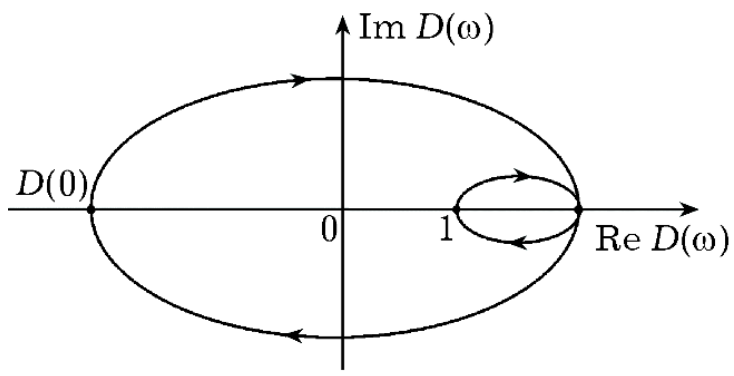

(b)

Fig. 4. Behavior of the function $D(\omega)$ in the traversal of the upper half-plane for $\mu<0$.

For $\alpha \ll 1$, we can obtain an approximate value of the smallest eigenvalue $\eta$ of the boundary-value problem (12)-(14): $\eta \approx p_{0}+b_{0} \alpha / p_{0}$. In this case, the quantity $\tau=-1 / \eta$, which characterizes the lifetime of the stratum, is much larger than the relaxation times $\tau_{\lambda}$ and $\tau_{v}$, and the stationary inhomogeneous distributions of ferroparticles in the magnetizable fluid can exist for a fairly long time.

\section{Conclusions}

We investigate inhomogeneous distributions of aggregates of ferroparticles that form in fixed layers of a magnetizable fluid under the action of a homogeneous external magnetic field. Equations that describe the change in the magnetic state of the fluid determine the КИ-system, in which the mean number of particles in aggregates is a fast variable, and the parameter of the effective magnetic field is a slow variable. We analyze the stationary solutions of these equation of the type of wide isolated and periodic strata and investigate their stability with respect to small perturbations by the methods of the theory of autosolitons. It is shown that, in small volumes of the fluid, i.e., under the condition $D_{v} \ll \ell^{2} \ll D_{\lambda}$, strata are unstable but can exist for a fairly long time. Under the action of perturbations, the breakdown of wide strata into strata of smaller sizes, in particular, into narrow strata, which exist at the same values of the magnetic field strength, but, as shown in [9], are unstable, is possible. In this case, strip structures of ferroparticles, which are similar to those observed in experiments with films of magnetizable fluids, form. 


\section{REFERENCES}

1. Yu. I. Dikanskii, "Experimental investigation of effective magnetic fields in a magnetic fluid," Magn. Gidrodinam., No. 2, 33-36 (1982).

2. A. Yu. Zubarev and L. Yu. Iskakova, "On the theory of phase transitions in magnetic fluids," Zh. Éksp. Teor. Fiz., 132, Issue 5, 1160-1172 (2007).

3. E. Kamke, Differentialgleichungen: Losungsmethoden und Losungen, Vol. I: Gewöhnliche Differentialgleichungen, B. G. Teubner, Leipzig (1977).

4. B. S. Kerner and V. V. Osipov, Autosolitons. A New Approach to Problems of Self-Organization and Turbulence, Kluwer, Dordrecht-Boston (1994).

5. L. D. Landau and E. M. Lifshitz, Quantum Mechanics. Non-Relativistic Theory [in Russian], Nauka, Moscow (1974).

6. N. F. Patsegon, "Some processes of self-organization in magnetizable media," Magn. Gidrodinam., No. 1, 13-23 (1993).

7. N. F. Patsegon and L. N. Popova, "Autowave processes in a magnetic fluid," Prikl. Gidromekh., 9, No. 1, 54-65 (2006).

8. N. F. Patsegon and L. N. Popova, "On the equilibrium magnetization of a magnetic fluid," Magn. Gidrodinam., 32, No. 3, 323-330 (1996).

9. N. F. Patsegon and L. N. Popova, "Formation and stability of spatial structures in a magnetic fluid," Prikl. Gidromekh., 5(77), No. 1, 62-70 (2003).

10. N. F. Patsegon and L. N. Popova, "Static autosolitons in a magnetic fluid," Visn. Kharkiv. Univ. Mat. Prykl. Mat. Mekh., Issue 4, No. 645, 93-101 (2004).

11. N. F. Patsegon and L. N. Popova, "Stationary structures in a magnetic fluid," Visn. Kharkiv. Nats. Univ. Mat. Model. Inform. Tehnol. Avtomat. Syst. Upravl., Issue 10, No. 833, 206-221 (2008).

12. N. F. Patsegon, L. N. Popova, and S. A. Sviridenko, "Qualitative investigation of structurization processes of a magnetic fluid," Visn. Kharkiv. Univ. Mat. Prykl. Mat. Mekh., Issue 52, No. 582, 81-89 (2003).

13. C. P. Pool and F. G. Owens, Introduction to Nanotechnology, John Wiley \& Sons, Hoboken (2003).

14. R. E. Rosensweig, Ferrohydrodynamics, Cambridge University Press, Cambridge (1985).

15. V. V. Chekanov, V. I. Drozdova, P. V. Nutsubidze, T. V. Skrobotova, and A. V. Cheremushkina, "Change in the magnetization of a magnetic fluid with change in aggregates," Magn. Gidrodinam., No. 1,3-9 (1984).

16. J. Chen, X. Chen, and S. Pu, "Realization of optical limiting with a magnetic fluid film," Opt. Commun., 276, No. 2, 268-271 (2007).

17. C. Y. Hong, I. J. Jang, H. E. Horng, C. J. Hsu, Y. D. Yao, and H. C. Yang, “Ordered structures in $\mathrm{Fe}_{3} \mathrm{O}_{4}$ kerosene-based ferrofluids," J. Appl. Phys., 81, No. 8, 4275-4277 (1997).

18. A. O. Ivanov, "Aggregates in magnetic fluids: droplets, chains and fractal clusters," in: Abstracts of the 8th Int. Conf. on Magnetic Fluids (June 29-July 3, 1998, Timisoara, Romania), Timisoara (1998), pp. 262-263.

19. J. Li, X.-D. Lin, Y.-Q. Lin, Y. Huang, and L. Bai, "Relaxation behavior measuring of transmitted light through ferrofluids films," Appl. Phys. B., 82, No. 1, 81-84 (2006).

20. B. Berkovski (editor), Magnetic Fluids and Application. Handbook, Begell House, New York (1996).

21. N. F. Patsegon and L. N. Popova, "Wave structures in magnetic fluids," Int. J. Fluid Mech. Res., 30, No. 3, $323-336$ (2003).

22. S.-Y. Yang, H.-E. Horng, C.-Y. Hong, "Structures, optical properties and potentially electro-optical applications of magnetic fluid films," Tamkang J. Sci. Eng., 5, No. 2, 85-93 (2002).

23. M. Zahn, "Magnetic fluid and nanoparticle applications to nanotechnology," J. Nanoparticle Res., 3, No. 1, 73-78 (2001). 